\title{
Mn-catalyzed oxydation of heavy oil in porous media: kinetics and some aspects of mechanism
}

Andrey Galukhin, ${ }^{\mathrm{a}, \mathrm{b} *}$ Mohammed Khelkhal, ${ }^{\mathrm{a}, \mathrm{b}}$ Alexander Gerasimov, ${ }^{\mathrm{a}}$ Timur Biktagirov, ${ }^{\mathrm{b}, \mathrm{c}}$ Marat Gafurov, ${ }^{\mathrm{c}}$ Alexander Rodionov, ${ }^{\mathrm{c}}$ Sergei Orlinskii ${ }^{\mathrm{c}}$

Kazan Federal University, 18 Kremlevskaya str., Kazan 420008, Russian Federation

${ }^{\mathrm{a}}$ Institute of Chemistry, Physical chemistry department

${ }^{\mathrm{b}}$ Institute of Geology and Petroleum Technologies

${ }^{\mathrm{c}}$ Institute of Physics

*Corresponding author. E-mail address: and_galuhin@mail.ru

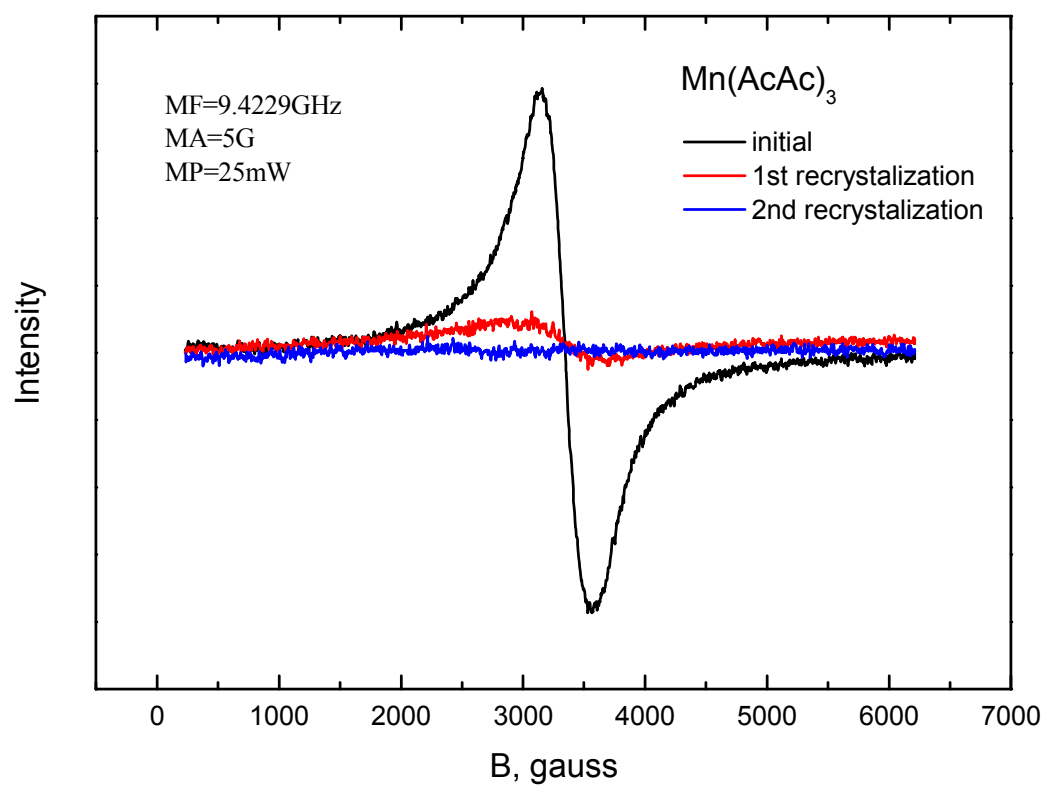

Figure 1. Control on purity of the $\mathrm{Mn}(\mathrm{AcAc})_{3}$ after recrystallization.

Table 1. DSC reaction intervals and the heat of reactions

\begin{tabular}{cccc}
\hline Sample & $\beta, \mathrm{K} \times \min ^{-1}$ & Interval, ${ }^{\circ} \mathrm{C}$ & $\begin{array}{c}\text { Heat of } \\
\text { reaction } \\
\left({ }_{\Delta} \mathrm{H}\right), \\
\mathrm{J} / \mathrm{g}\end{array}$ \\
\hline \multirow{3}{*}{ Non-catalytic } & 10 & $289-547$ & 10434 \\
& 15 & $301-561$ & 9523 \\
& 20 & $309-571$ & 9008
\end{tabular}




\begin{tabular}{cccc} 
& 5 & $280-518$ & 10844 \\
Catalytic & 10 & $289-543$ & 9356 \\
& 15 & $302-560$ & 9178 \\
& 20 & $307-572$ & 8981 \\
\hline
\end{tabular}

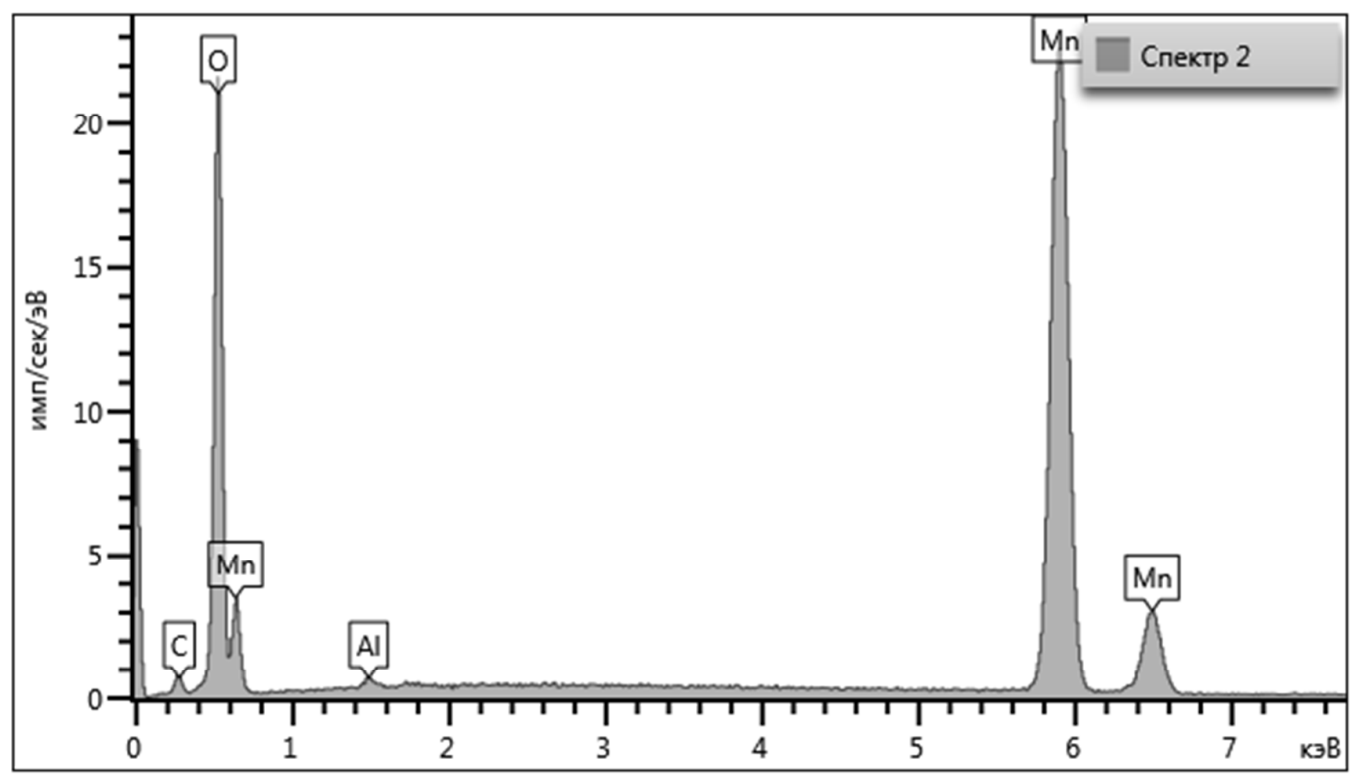

Figure 2. EDX spectrum of $\mathrm{Mn}_{2} \mathrm{O}_{3}$ nanoparticles.

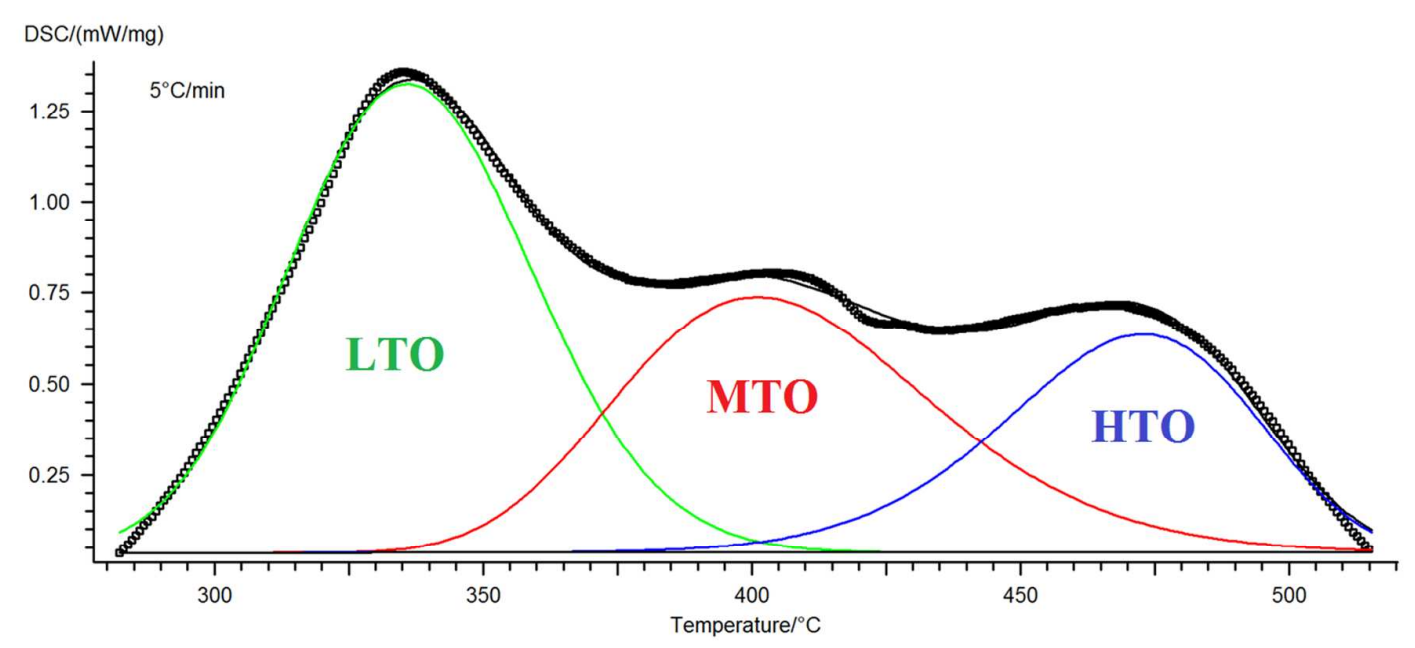

Figure 3. Peaks separation of DSC curve for $\mathrm{Mn}-\mathrm{Al}_{2} \mathrm{O}_{3}$-oil sample $\left(5^{\circ} \mathrm{C} / \mathrm{min}\right)$. 


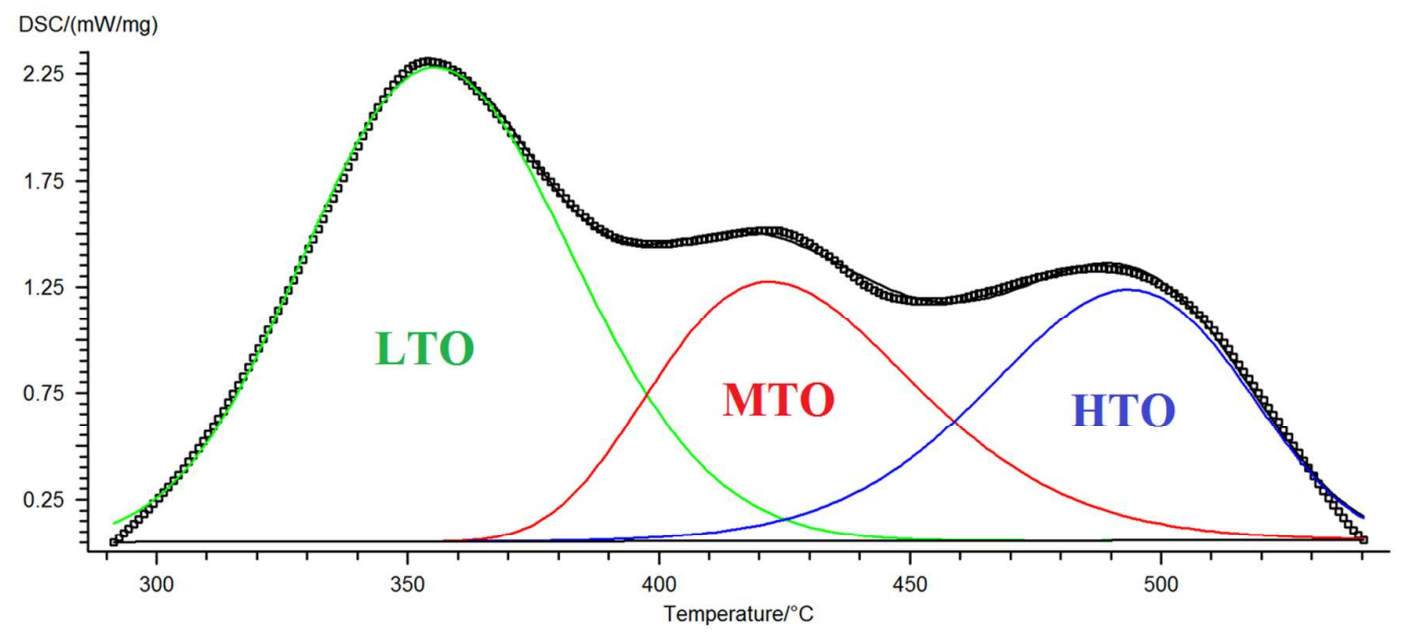

Figure 4. Peaks separation of DSC curve for $\mathrm{Mn}-\mathrm{Al}_{2} \mathrm{O}_{3}$-oil sample $\left(10{ }^{\circ} \mathrm{C} / \mathrm{min}\right)$.

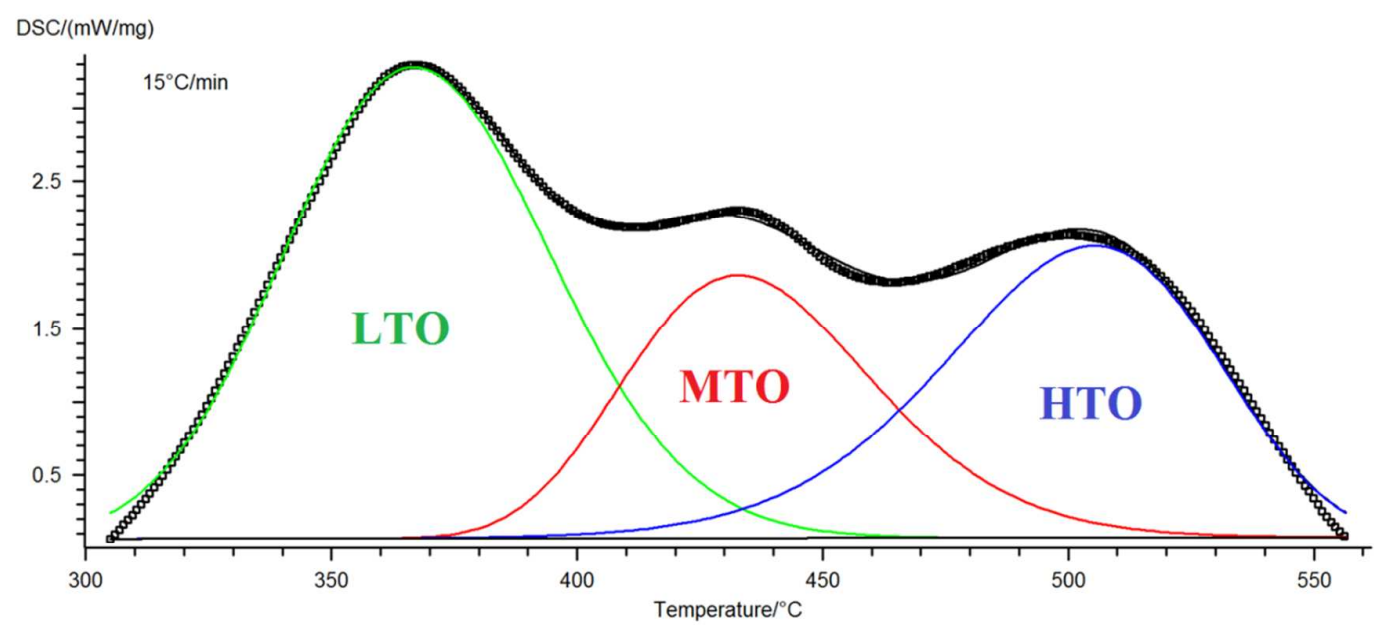

Figure 5. Peaks separation of DSC curve for $\mathrm{Mn}-\mathrm{Al}_{2} \mathrm{O}_{3}$-oil sample $\left(15^{\circ} \mathrm{C} / \mathrm{min}\right)$.

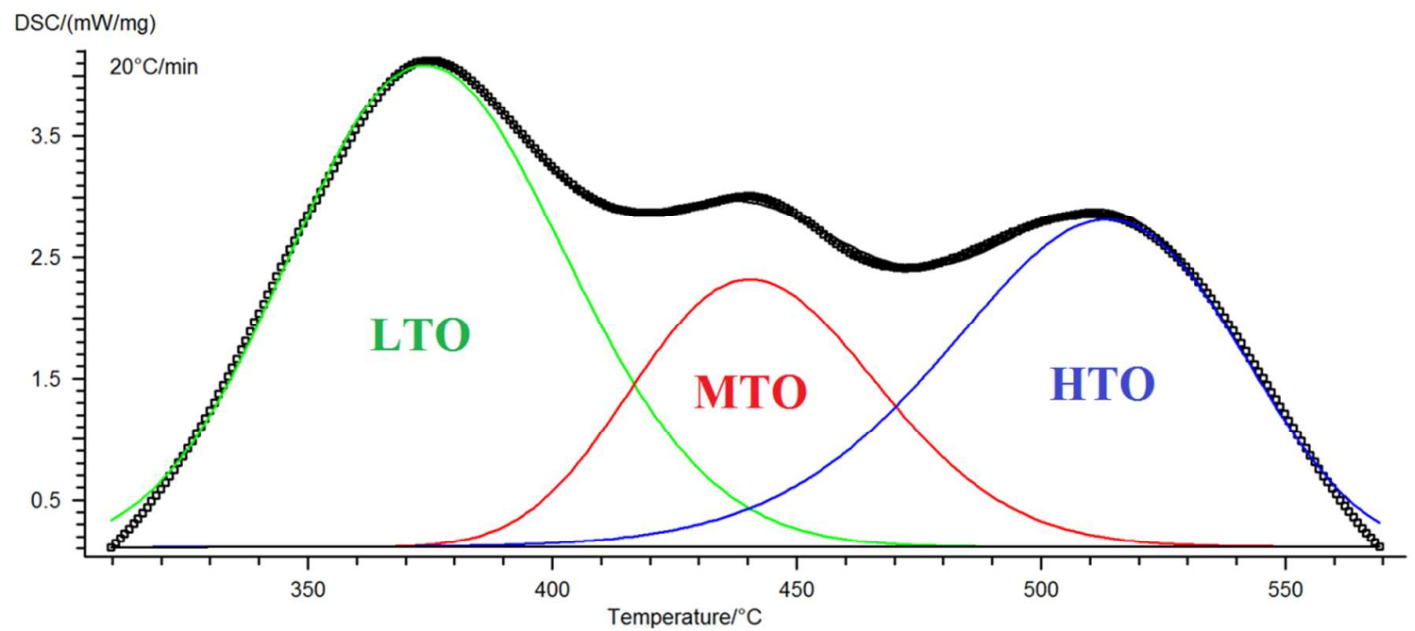

Figure 6. Peaks separation of DSC curve for $\mathrm{Mn}-\mathrm{Al}_{2} \mathrm{O}_{3}$-oil sample $\left(20^{\circ} \mathrm{C} / \mathrm{min}\right)$. 